\title{
PREVALÊNCIA DE ARTEFATOS EM EXAMES DE RESSONÂNCIA MAGNÉTICA DO ABDOME UTILIZANDO A SEOÜÊNCIA GRASE: COMPARÁVEL COM AS MELHORES SEOÜÊNCIAS RÁPIDAS?*
}

\author{
Viviane Vieira Francisco ${ }^{1}$, Giuseppe D'Ippolito ${ }^{2}$, Gláucia Palácio de Andrade e Silva ${ }^{3}$, \\ Alexandre Sérgio de Araújo Bezerra ${ }^{4}$, Jacob Szejnfeld ${ }^{5}$
}

Resumo OBJETIVO: Determinar a freqüência global de artefatos na seqüência "gradient and spin echo" (GRASE), por tipo e grau do artefato, em exames de ressonância magnética de abdome; realizar comparação entre as seqüências GRASE e duas seqüências TSE previamente selecionadas como aquelas com melhor relação sinalruído e menor incidência de artefatos. MATERIAIS E MÉTODOS: Foi realizado estudo prospectivo, autopareado, em 86 pacientes submetidos a ressonância magnética de abdome superior, sendo adquiridas a seqüência GRASE com sincronizador respiratório e supressão de gordura e seis seqüências TSE ponderadas em T2. Dentre as seis seqüências TSE, foram previamente selecionadas aquelas com melhor relação sinalruído e menor número de artefatos, que foram as realizadas com supressão de gordura e com sincronizador respiratório, sendo uma com bobina de corpo (seqüência 1) e outra com bobina de sinergia (seqüência 2). A análise das imagens foi realizada por dois observadores em consenso, quanto a presença, grau e tipo de artefato. Posteriormente os dados foram analisados estatisticamente, através do teste de Friedman e do qui-quadrado. RESULTADOS: A freqüência absoluta de artefatos nas seqüências utilizadas foi de $65,02 \%$. Os artefatos mais encontrados nas três seqüências estudadas foram os de respiração (30\%) e de pulsação (33\%). Apenas $3 \%$ dos casos apresentaram algum tipo de artefato que dificultava a análise das imagens. As freqüências de artefatos nas diversas seqüências foram: GRASE, 67,2\%; seqüência TSE 1, 62,2\%; seqüência TSE 2, 65,5\%. Não houve diferença estatisticamente significante na freqüência de artefatos encontrados nas seqüências GRASE e nas seqüências TSE ( $p=0,845$; NS). CONCLUSÃO: As seqüências GRASE e TSE ponderadas em T2 com sincronizador respiratório e com supressão de gordura, independentemente da bobina utilizada, apresentam freqüentemente artefatos, porém com incidência semelhante e geralmente sem interferência na avaliação das imagens.

Unitermos: Artefato; Ressonância magnética; Abdome.

Abstract Prevalence of artifacts in abdominal magnetic resonance imaging using GRASE sequence: a comparison with TSE sequences.

OBJECTIVE: To determine the overall frequency of artifacts per type and grade using the GRASE sequence in abdominal magnetic resonance; to compare GRASE sequences with two previously selected TSE sequences as well as sequences with best signal-noise ratio and lower incidence of artifacts. MATERIALS AND METHODS: A prospective self-paired study was carried out in $\mathbf{8 6}$ patients submitted to upper abdominal magnetic resonance using a GRASE sequence obtained upon respiratory triggered and fat suppression and six TSE T2-weighted sequences. Among the six TSE sequences, those bearing the best signal-noise ratio and lower number of artifacts were previously selected, which consisted of those performed with fat suppression and respiratory triggering: one using a conventional body coil (sequence 1) and a second sequence using a synergy coil (sequence 2). Image analysis was carried out by two observers upon consensus regarding the presence, grade and type of artifact thereon. Subsequently, data were statistically analyzed using the Friedman test and chi-square. RESULTS: The absolute frequency of artifacts in all sequences was $65.02 \%$. Most common artifacts in the three sequences analyzed were breathing $(30 \%)$ and pulsation $(33 \%)$ artifacts. Only in $3 \%$ of the cases artifacts interfered with the analysis of the images. The frequency of artifacts in the different sequences was: GRASE, $67.2 \%$; TSE sequence 1, 62.2\%; TSE sequence 2, $65.5 \%$. There was no significant statistical difference between artifact frequency seen with GRASE and TSE sequences ( $p=0.845$; NS). CONCLUSION: GRASE and TSE T2-weighted, respiratory triggered, fat suppressed sequences often produce artifacts, notwithstanding the coil, although, with similar frequency and generally without interfering with the evaluation of the images.

Key words: Artifact; Magnetic resonance; Abdomen.

* Trabalho realizado no Departamento de Diagnóstico por Imagem (DDI) da Universidade Federal de São Paulo/Escola Paulista de Medicina (Unifesp/EPM), São Paulo, SP.

1. Médica Pós-graduanda do DDI-Unifesp/EPM.

2. Professor do DDI-Unifesp/EPM.
3. Doutora em Radiologia Clínica do DDI-Unifesp/EPM. 4. Mestre em Radiologia Clínica do DDI-Unifesp/EPM. 5. Professor Livre-Docente, Chefe do DDI-Unifesp/EPM. Endereço para correspondência: Prof. Dr. Giuseppe D'Ippolito. Rua Professor Filadelfo Azevedo, 617, ap. 61, Vila Nova Con- ceição. São Paulo, SP, 04508-011. E-mail: giuseppe_dr@uol. com.br

Recebido para publicação em 28/10/2004. Aceito, após revisão, em 15/12/2004. 


\section{INTRODUÇÃo}

Durante a última década, a ressonância magnética (RM) vem apresentando rápida evolução na qualidade das imagens, em razão do surgimento de novas sequiências e dos avanços técnicos dos novos equipamentos, tendo sido observada redução significativa no tempo de aquisição das imagens e melhora nos parâmetros de obtenção das sequiências ${ }^{(1)}$.

Entre os progressos tecnológicos destacam-se o surgimento de equipamentos com gradientes mais potentes e eficientes que permitiram a criação de sequiências de pulso mais rápidas, reduziram o tempo de exame e minimizaram os artefatos de movimento respiratórios e peristálticos ${ }^{(2)}$. Os exames de RM de abdome se beneficiaram particularmente destes avanços, ampliando a sua utilização.
Entre as principais aplicações da RM do abdome destaca-se o estudo de lesões hepáticas focais $^{(2,3)}$, quando as sequiências ponderadas em T2 têm papel relevante, demonstrando alta sensibilidade e especificidade na detecção e caracterização dessas lesões ${ }^{(2)}$.

Técnicas rápidas e ultra-rápidas de aquisição das imagens ponderadas em T2 permitiram superar as principais limitações das sequiências spin-eco decorrentes de artefatos de movimento ${ }^{(\mathbf{3 , 4})}$. Entre essas técnicas destacam-se a "fast field echo" (FFE) ou FLASH, a "turbo spin echo" (TSE) e a "half fourier single shot" (HASTE) ${ }^{(3)}$.

Nesse contexto, foi desenvolvida a sequiência "gradient and spin echo" (GRASE), que é uma sequiência híbrida gradiente-eco com um tempo de aquisição menor que as TSE ponderadas em T2 e com elevado contraste entre partes moles ${ }^{(5)}$ (Figura 1).
Apesar disso, existem resultados conflitantes quanto à aplicação clínica da seqüência GRASE em relação às sequiências TSE ponderadas em T2, talvez relacionados à maior ocorrência de artefatos na seqüência GRASE ${ }^{(1,6)}$.

Portanto, os objetivos deste trabalho foram: determinar a frequiência de artefatos nas sequiências GRASE por grau e tipo de artefato em exames de RM do abdome superior, e comparar a freqüência de artefatos presentes nas sequiências GRASE e TSE ponderadas em T2, classificando-os por tipo e grau.

\section{MATERIAIS E MÉTODOS}

Este é um estudo prospectivo, observacional, transversal e autopareado realizado no Departamento de Diagnóstico por Imagem da Universidade Federal de São

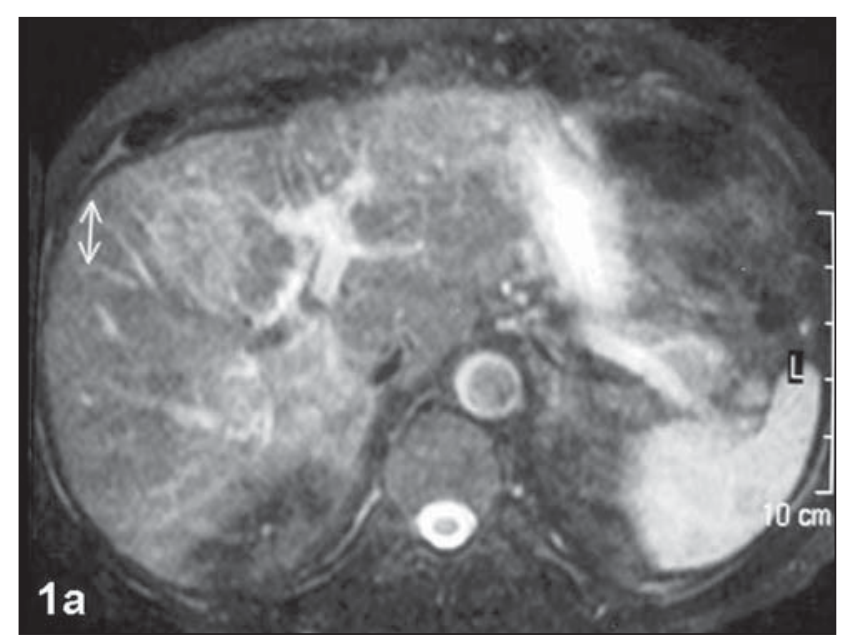

Figura 1. Imagens ponderadas em $T 2$ de um mesmo paciente demonstram a identificação de metástases hepáticas na seqüência TSE (1a e 1b) e na seqüência GRASE (1c). Na seqüência GRASE pode-se observar ótimo contraste entre as lesões e o parênquima hepático e maior conspicuidade das lesões.
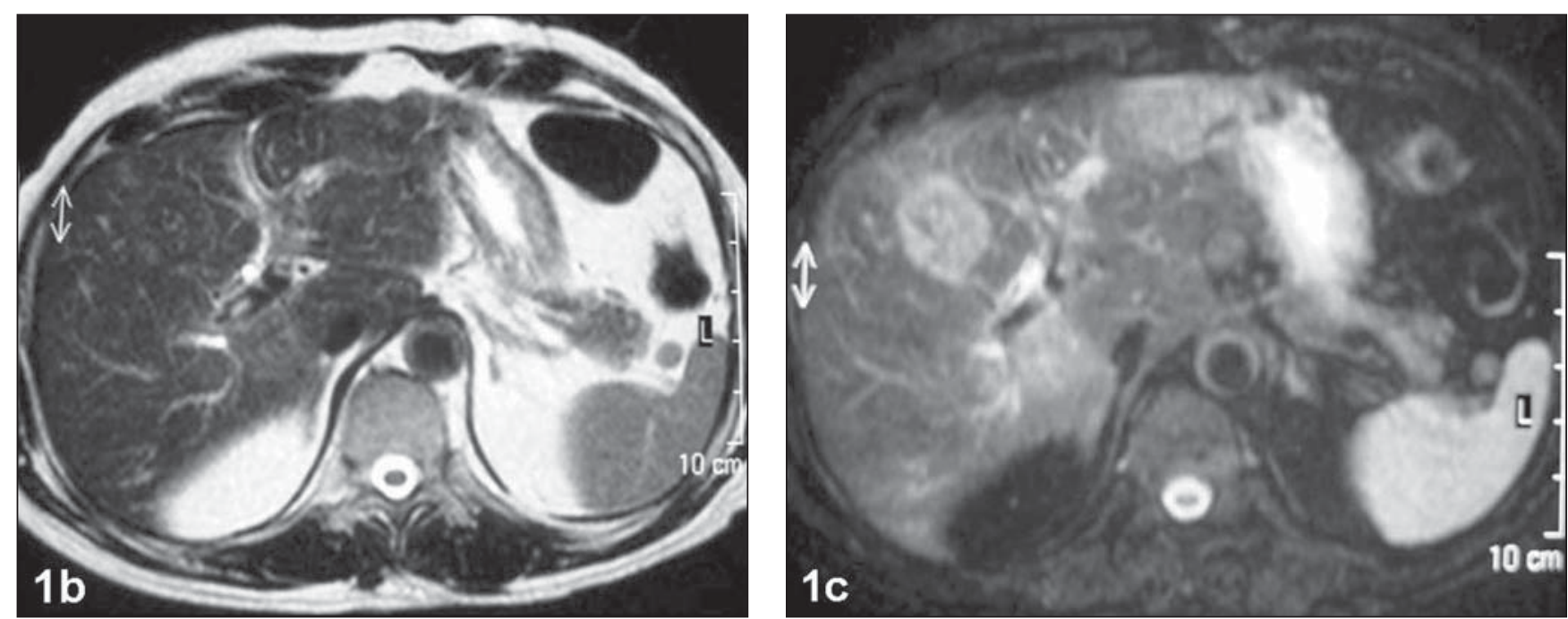
Paulo/Escola Paulista de Medicina (Unifesp/EPM), no qual foram examinados 86 pacientes (44 homens e 42 mulheres) com idade variando entre 18 e 82 anos (média de 50 anos, mediana de 56 anos), encaminhados para a realização de RM de abdome superior, pelas mais diversas indicações, no período de janeiro de 2000 a dezembro de 2001.

O protocolo de pesquisa foi aprovado pelo Comitê de Ética em Pesquisa da Unifesp/EPM e os pacientes assinaram termo de consentimento informado.

Foram incluídos os pacientes maiores de 18 anos, independentemente da indicação do exame, da doença de base, do ritmo respiratório ou da sua capacidade de manter apnéia.

Os critérios de exclusão foram: necessidade de uso de sedação anestésica para realizar o exame (em pacientes claustrofóbicos, por exemplo) e pacientes inconscientes ou incapazes de compreensão.

Os exames foram realizados em aparelho da marca Philips, modelo ACS NT 15, operando com alto campo magnético $(1,5$ T) e equipado com gradientes de $15 \mathrm{mT} / \mathrm{s}$. Na primeira fase do estudo foram adquiridas sete diferentes sequiências, sendo seis seqüências TSE ponderadas em T2 e a seqüência GRASE. As seqüências TSE dife- riam quanto a: presença ou ausência de supressão de gordura; técnica de controle do artefato de respiração (sincronizador respiratório ou apnéia); e bobina utilizada (bobina de corpo ou bobina de sinergia). A seqüência GRASE foi realizada com sincronizador respiratório, sem supressão de gordura e com bobina de corpo.

Dentre as seis sequiências TSE, foram selecionadas as duas melhores seqüências quanto à menor prevalência de artefatos e aquelas que apresentavam a melhor relação sinal-ruído, conforme publicado em estudo anterior ${ }^{(7)}$. As características destas seqüências estão detalhadas no Quadro 1.

Em todos os pacientes foi utilizada faixa de compressão abdominal, para reduzir artefatos de respiração, e feita injeção endovenosa de antiespasmódico (brometo de nbutilescopolamina, $2 \mathrm{ml}, 40 \mathrm{mg}$ ), imediatamente antes do início do exame, com o intuito de reduzir os artefatos causados pelos movimentos peristálticos das alças intestinais.

A análise das imagens foi realizada por dois observadores em consenso, com pelo menos três anos de experiência em RM do abdome.

A interpretação de cada seqüência foi realizada com uma semana de intervalo e foi analisada a presença ou ausência de ar- tefatos, o tipo de artefato e quando estes estavam presentes, se interferiram ou não na análise das imagens.

Os artefatos foram classificados em: respiração, pulsação, peristaltismo, suscetibilidade magnética, fantasma e "chemical shift", segundo suas características já estabelecidas na literatura e transmitidas aos observadores $^{(\mathbf{6 - 9})}$ (Figuras 2 a 6).

Os artefatos observados foram também graduados em graus 1 e 2, em função de seu impacto na qualidade da imagem e na sua interferência na interpretação destas: grau 1 - os artefatos não interferem na interpretação das imagens (Figura 7); grau 2 - os artefatos interferem na interpretação das imagens (Figura 8).

A amostra recebeu tratamento estatístico através do teste de Friedman, de acordo com a prevalência de cada artefato para as três seqüências. Foi calculada a prevalência global de artefatos, por tipo e por seqüência analisada, procurando estabelecer a ocorrência de diferenças estatísticas por meio do teste do qui-quadrado $\left(\chi^{2}\right)$. Em todos os cálculos o valor de significância para rejeição da hipótese de nulidade foi fixado em um valor igual ou menor que $5 \%$ ( $\mathrm{p} \leq 0,05)$. Quando a estatística calculada foi significante, usamos um asterisco $(*)$ para caracterizá-la. Caso contrário, usamos a sigla NS (não significante).

\section{RESULTADOS}

A frequiência total de artefatos encontrados nas três sequiências analisadas foi de $65,02 \%$.

As seqüências 2, 1 e GRASE apresentaram freqüência global de artefatos de $62,2 \%, 65,5 \%$ e $67,2 \%$, respectivamente. $\mathrm{O} \chi^{2}$ calculado foi de 0,336 e a diferença entre as sequiências não foi estatisticamente significante $(\mathrm{p}=0,845)$.

Os artefatos mais comumente encontrados nas três sequiências foram: de pulsação em 33\% dos casos, respiratórios em 30\% dos casos, de peristaltismo em $15 \%$ dos casos, fantasmas em $7 \%$ dos casos, "chemical shift" em $13 \%$ dos casos e suscetibilidade magnética em $2 \%$ dos casos.

Considerando-se o grau do artefato, $97 \%$ não interferiam na interpretação das imagens e apenas $3 \%$ prejudicavam a análise dos exames.
TSE, turbo spin eco; BC, bobina de corpo; SPIR, com supressão de gordura; SR, sincronização respiratória; SYN, bobina de sinergia; GRASE, "gradient and spin echo"; TR, tempo de repetição; TE, tempo de eco; FOV, "field of view"; RFOV, "rectangular field of view". 


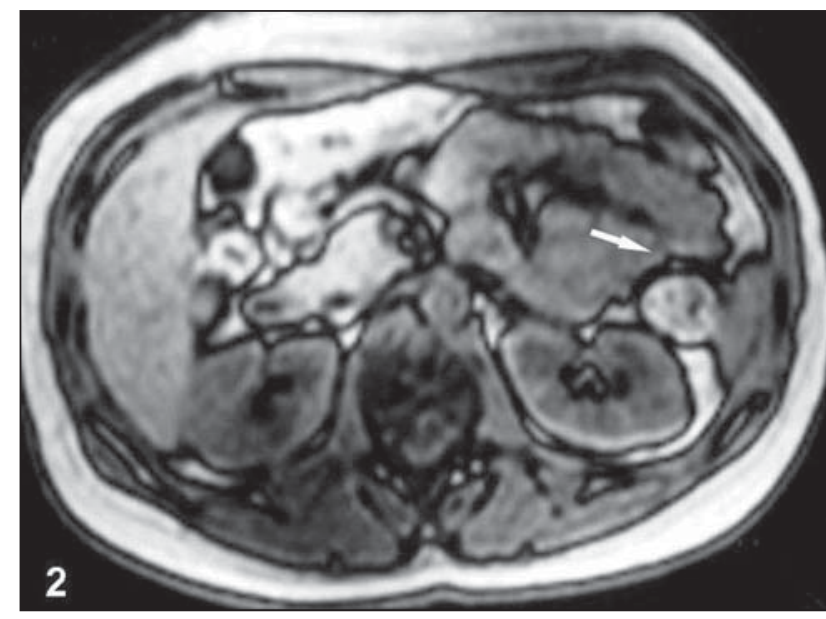

Figura 2. Artefato "chemical shift". Nota-se fina linha hipointensa ao redor das vísceras (seta).

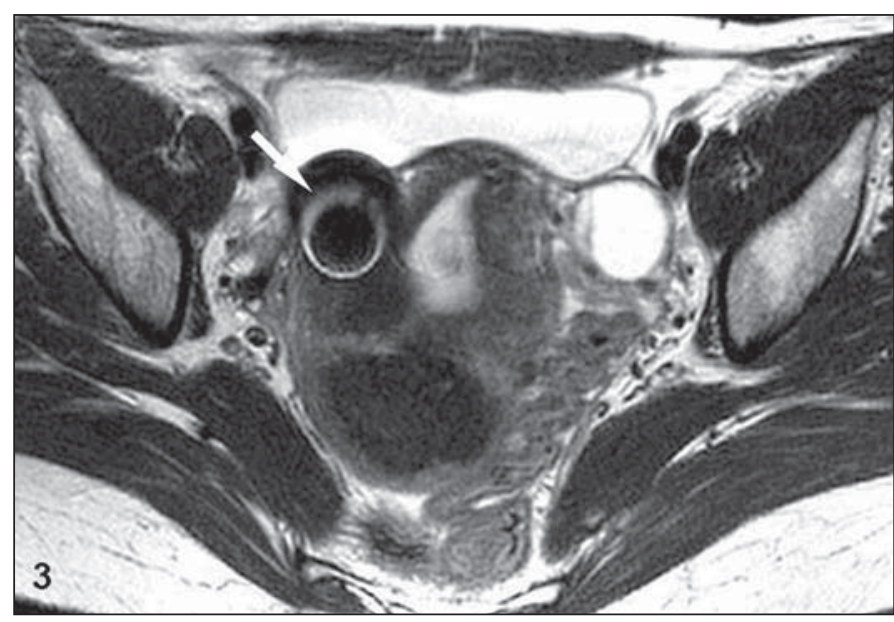

Figura 3. Artefato de suscetibilidade magnética (material metálico) na fossa ilíaca direita (seta) pós-manipulação cirúrgica.
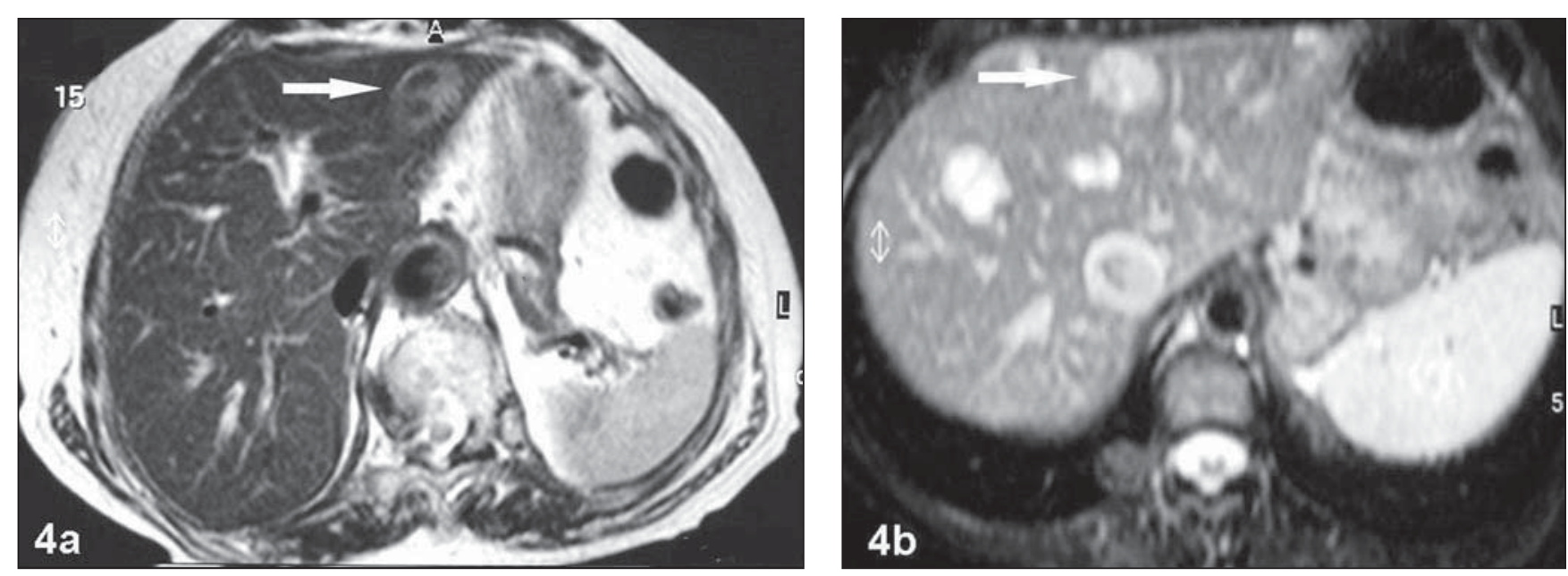

Figura 4. Artefatos de pulsação da aorta (4a) e veia cava inferior (4b) (setas), simulando nódulos hepáticos.

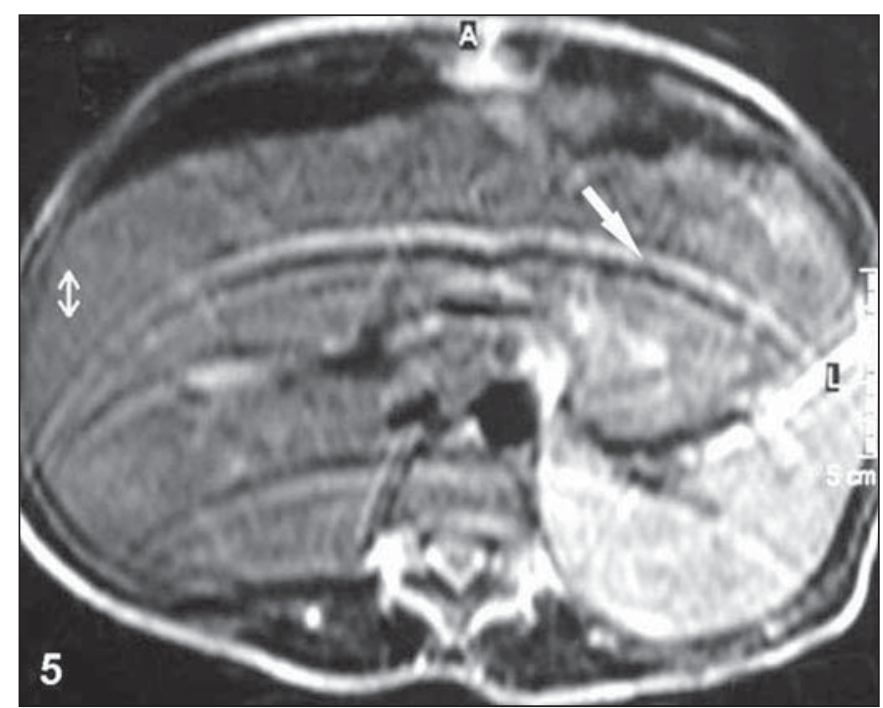

Figura 5. Artefatos de respiração (seta).

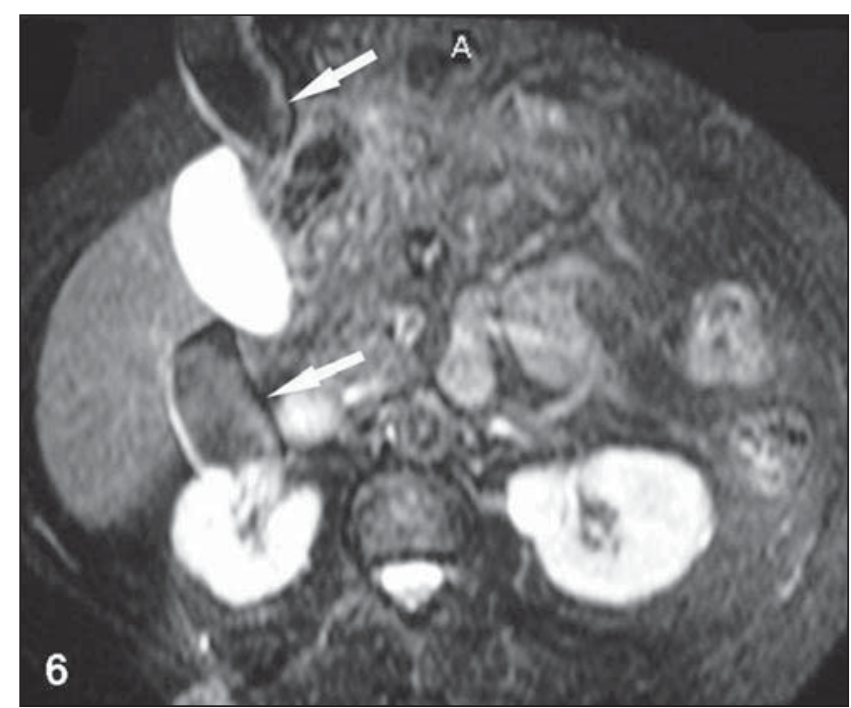

Figura 6. Artefato tipo fantasma da vesícula biliar (setas). 


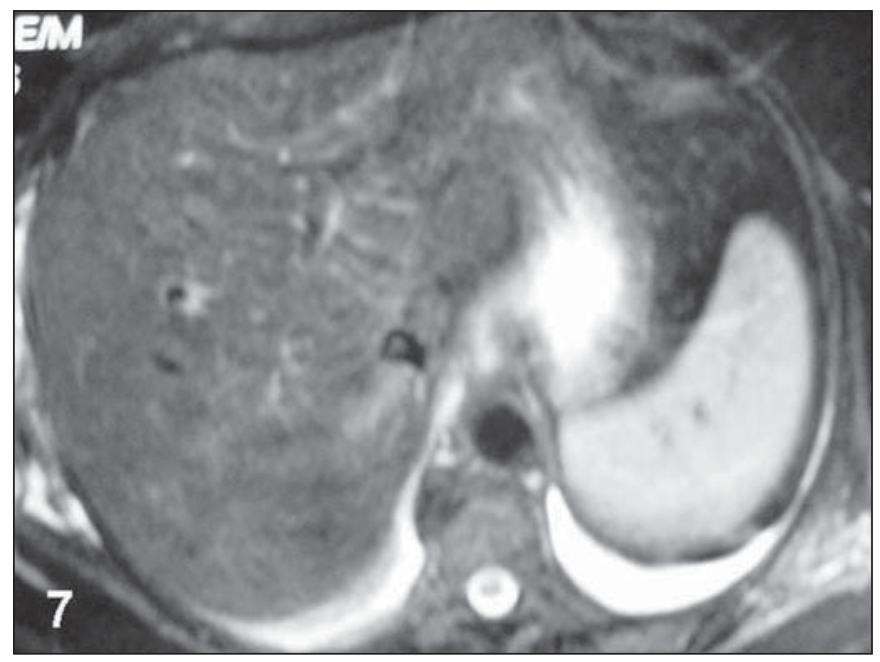

Figura 7. Artefato de respiração grau 1. Não interfere na interpretação da imagem.

$\mathrm{Na}$ análise do comportamento de cada tipo de artefato entre as três sequiências, apenas o de respiração $\left(\chi^{2}=13,339 ; \mathrm{p}<\right.$ $0,001)$ e o "chemical shift" $\left(\chi^{2}=9,579 ; \mathrm{p}\right.$ $=0,0008)$ apresentaram diferença estatisticamente significante .

Os artefatos mais freqüentemente encontrados para as três seqüências utilizadas estão demonstrados na Tabela 1.

\section{DISCUSSÃO}

Entre as inúmeras sequiências de RM disponíveis para avaliação do fígado e órgãos abdominais, as imagens ponderadas em T2 fornecem as mais importantes informações sobre a detecção e caracterização dos nódulos hepáticos ${ }^{(\mathbf{1 , 8 , 9 )}}$. A aquisição de imagens no abdome, com elevada resolução espacial e temporal, tem sido, nos últimos anos, importante área de interesse de investigação científica ${ }^{(\mathbf{1}-\mathbf{6})}$. Assim, vêm sendo desenvolvidas novas seqüências, bem como progressos nas técnicas de obtenção das imagens, particularmente quanto ao uso de métodos para controle de artefatos de respiração, supressão de gordura e tipo de bobina de radiofrequiência.

Neste campo de pesquisa, a seqüência GRASE surgiu como uma interessante alternativa para as técnicas TSE, em virtude do curto tempo de aquisição das imagens, da alta resolução de contraste e da boa qualidade de imagens. No entanto, ainda não existe pleno consenso na literatura com relação à aplicabilidade clínica da seqüên-

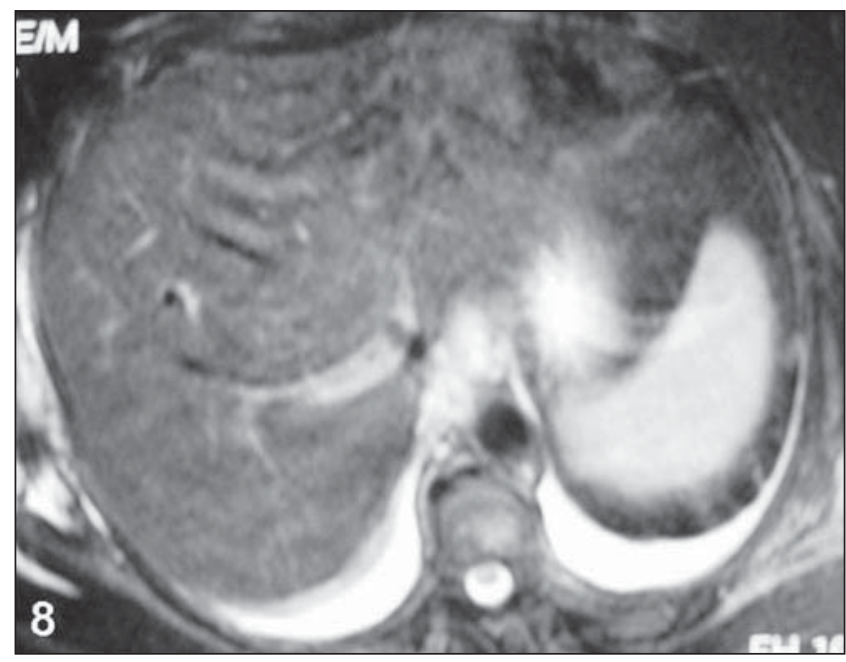

Figura 8. Artefato de respiração grau 2. Interfere na interpretação da imagem.

Tabela 1 Prevalência de cada tipo de artefato nas seqüências analisadas.

\begin{tabular}{|l|c|c|c|}
\hline Artefatos & $\begin{array}{c}\text { Seqüência 1 } \\
\text { (TSE/BC/SPIR/SR) }\end{array}$ & $\begin{array}{c}\text { Seqüência 2 } \\
\text { (TSE/SYN/SPIR/SR) }\end{array}$ & GRASE \\
\hline Pulsação & $63,15 \%$ & 45,0 & 46,3 \\
Respiração & $21,00 \%$ & 57,5 & 56,1 \\
Fantasma & $13,10 \%$ & 7,5 & 9,7 \\
Peristaltismo & $31,60 \%$ & 20,0 & 14,6 \\
"Chemical shift" & $10,50 \%$ & 12,5 & 34,1 \\
Suscetibilidade & $2,60 \%$ & 2,5 & 2,4 \\
\hline
\end{tabular}

TSE, turbo spin-eco; BC, bobina de corpo; SPIR, supressão de gordura; SR, sincronizador respiratório; SYN, bobina de sinergia; GRASE, "gradient and spin echo".

cia GRASE, devido ao pequeno número de trabalhos realizados e à variabilidade técnica de aquisição implementada ${ }^{(2,3,6)}$.

Contudo, existem evidências de que a seqüência GRASE apresenta elevada eficácia para detecção e caracterização de lesões hepáticas focais no fígado ${ }^{(2)}$. Choe $e t$ $a l .{ }^{(3)}$ demonstraram menor variabilidade interobservador na diferenciação de lesões benignas e malignas no fígado com a técnica GRASE em relação às TSE.

Na revisão bibliográfica realizada destacam-se poucos trabalhos prospectivos, com casuísticas heterogêneas, parâmetros de inclusão e exclusão pouco definidos e grande variabilidade de parâmetros técnicos utilizados $(\mathbf{2 , 3 , 1 0})$.

Não foram encontrados trabalhos na literatura que tenham estudado os artefatos presentes na seqüência GRASE.

No presente estudo encontrou-se prevalência absoluta de artefatos na sequiência GRASE maior que nas duas seqüências
TSE, mas esta diferença foi pequena e estatisticamente não significante. Os artefatos observados foram freqüentes $(65,02 \%)$, porém a maioria deles $(97 \%)$ não interferiu na análise das imagens.

Neste trabalho a utilização da sequiência GRASE foi responsável por $56,1 \%$ de artefatos de respiração, $46,3 \%$ de artefatos de pulsação, 34,1\% de "chemical shift", $14,6 \%$ de peristaltismo, $9,7 \%$ de fantasmas e $2,4 \%$ de suscetibilidade. No estudo de Pijl et al. ${ }^{(1)}$, os autores verificaram que a sequiência GRASE foi bastante suscetível a artefatos de movimento. Esse resultado poderia ter sido decorrente do fato de as imagens terem sido obtidas sem o uso do sincronizador respiratório. Porém, no nosso trabalho, utilizamos de rotina o sincronizador respiratório na seqüência GRASE e ainda assim os artefatos de movimento foram bastante freqüentes.

Foi descrito que a seqüência GRASE poderia apresentar uma maior prevalência 
do artefato de suscetibilidade magnética sobre as demais seqüências. Isso poderia acontecer devido à perda momentânea da homogeneidade do campo durante a aquisição das imagens e da magnetização de tecidos com alto potencial magnético, como o cálcio, o meio de contraste, a gordura e metais $^{(4)}$. Foi encontrada baixa prevalência deste artefato no presente estudo, que pode ter ocorrido devido às características inerentes dos pacientes estudados ou pela técnica adequada de aquisição das imagens.

Neste trabalho, foi encontrado grande predomínio dos artefatos "chemical shift" na seqüência GRASE sobre os outros artefatos estudados, e apenas este demonstrou ser estatisticamente significante (Tabela 1). Este artefato é decorrente de alterações do sinal que resultam das diferenças de freqüiência de precessão (freqüência de ressonância) dos prótons de hidrogênio presentes nas moléculas de água e lipídios (Figura 2) ${ }^{(\mathbf{1 1})}$. Apesar de frequiente, este artefato não interfere na interpretação das imagens, e em algumas situações clínicas pode até contribuir para esclarecimento diagnóstico ${ }^{(\mathbf{1 1})}$. A maior freqüência encontrada deste artefato pode ser devida à pouca homogeneidade do campo, que pode acontecer em decorrência dos múltiplos pulsos de radiofreqüência durante a aquisição de imagens na técnica GRASE. A utilização de voxel menor e o aumento da largura da banda poderiam minimizar esse tipo de artefato. Além disso, é possível melhorar a técnica de aquisição desta seqüência com os seguintes recursos: dividindo-se o trem de eco; obtendo-se duas aquisições em tempo de eco e repetição diferentes; utilizando-se diferentes tempos de eco e repetição para obtenção de imagens distintas; aumentando-se o volume em três dimensões; utilizando-se matriz de alta resolução ${ }^{(11,12)}$.

$\mathrm{O}$ artefato de pulsação foi o mais freqüente na análise global das três seqüências, porém foi estudado separadamente do artefato tipo fantasma, ao contrário de alguns autores, que analisam a pulsação como um artefato de movimento do tipo fantasma (Figura 4) ${ }^{(\mathbf{5})}$.

Segundo alguns trabalhos, a sequiência GRASE praticamente eliminaria os artefa- tos de respiração, devido ao tempo de aquisição de imagem ser menor que um ciclo respiratório. No entanto, este artefato foi o segundo mais comum, com prevalência semelhante à da seqüência 2 (TSE/SYN/

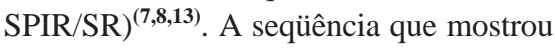
a menor prevalência de artefatos respiratórios foi a 1 (TSE/BC/SPIR/SR), mas a diferença entre as três não foi significativa.

A grande discrepância de resultados na literatura ocorre devido à variedade de parâmetros utilizados nas aquisições de imagem, a avaliação subjetiva da conspicuidade para lesões e a falta de estudos qualitativos para os artefatos ${ }^{(\mathbf{1 4 - 2 0})}$

Além disso, as novas sequiências que vêm sendo otimizadas e as características dos equipamentos disponíveis no mercado podem causar sensíveis diferenças nos resultados estatísticos ${ }^{(\mathbf{2 0})}$.

Esperamos que este estudo contribua para uma melhor elucidação das características da seqüência GRASE, dos artefatos mais prevalentes e da sua relação com as melhores seqüências rápidas TSE ponderadas em $\mathrm{T} 2$.

Concluindo, a partir da análise dos resultados obtidos do uso de três seqüências de imagem, ponderadas em T2, em estudos por RM do abdome, observou-se que as seqüências GRASE apresentaram freqüentemente artefatos, sem interferir na interpretação das imagens, e que a prevalência dos artefatos entre as três seqüências estudadas é semelhante e geralmente sem interferência na avaliação das imagens.

\section{REFERÊNCIAS}

1. Pijl MEJ, Wasser MNJM, van Persijn van Meerten EL, et al. Comparison of inversion-recovery gradient- and spin-echo and fast spin-echo techniques in the detection and characterization of liver lesions. Radiology 1998;209:427-434.

2. Karantanas AH, Papanikolau N. T2-weighted magnetic resonance imaging of the liver: comparison of fat-suppressed GRASE with conventional spin echo, fat-suppressed turbo spin echo, and gradient echo at 1.0 T. Abdom Imaging 2001;26:139-145.

3. Choe KA, Smith RC, Wilkens K, Constable T. Motion artifact in T2-weighted fast spin-echo images of the liver: effect on image contrast and reduction of artifact using respiratory triggering in normal volunteers. J Magn Reson Imaging 1997; 7: 298-302.

4. Mugler JP 3rd. Improved three-dimensional GRASE imaging with the SORT phase-encoding strategy. J Magn Reson Imaging 1999;9:604-612.
5. Feinberg DA, Oshio K. GRASE (gradient- and spinecho) MR imaging: a new fast clinical imaging technique. Radiology 1991;181:597-602.

6. Johnson G, Feinberg DA, Venkataraman V. A comparison of phase encoding ordering schemes in T2weighted GRASE imaging. Magn Reson Med 1996;36:427-435.

7. Palácio GAS, Francisco VV, Tiferes DA, D'Ippolito G, Szejnfeld J. Prevalência de artefatos em exames de ressonância magnética do abdome, comparando seis seqüências TSE ponderadas em T2. Radiol Bras 2002;35(Supl no 1):8.

8. Soyer P, Le Normand S, de Givry SC, Gueye C, Somveille E, Scherrer A. T2-weighted spin-echo MR imaging of the liver: breath-hold fast spin-echo versus non-breath-hold fast spin-echo images with and without fat suppression. AJR 1996;166:593597.

9. Soyer P, Gouhiri M, Rondeau Y, Spelle L, Mosnier $\mathrm{H}$, Scherrer A. Non-breath-hold fast spin-echo versus breath-hold fast spin-echo and spoiled gradientrecalled echo MR imaging in the detection of hepatic tumors: correlation with surgical findings. AJR 1997;168:1199-1204.

10. Ferrucci JT. Advances in abdominal MR imaging. RadioGraphics 1998;18:1569-1586.

11. Walter C, Jockenhofer A, Heindel W, et al. Fast and ultrafast magnetic resonance imaging in renal lesions. J Magn Reson Imaging 1998;8:1219-1227.

12. Palácio GAS, Francisco VV, Abbehusen CL, Tiferes DA, D'Ippolito G, Szejnfeld J. Artefatos em ressonância magnética de abdome: ensaio iconográfico. Radiol Bras 2002;35:371-376.

13. Jakobus RP, Rummeny EJ, Reimer et al. Rapid spin echo sequences for detection and characterization of focal liver lesions. Use of fast T2-weighted spin echo with fat suppression and fast spin echo with combined gradient echo (GRASE). Radiology 1995;35:911-918.

14. Arena L, Morehouse HT, Safir J. MR imaging artifacts that simulate disease: how to recognize and eliminate them. RadioGraphics1995;15:13731394.

15. Abbehusen CL, D'Ippolito G, Palácio GAS, Szejnfeld J. Estudo comparativo das sequiências rápidas ponderadas em T2, utilizando-se sincronização respiratória, apnéia, supressão de gordura, bobina de corpo e sinergia para avaliação do fígado pela ressonância magnética. Radiol Bras 2003;36:261272.

16. Wood ML, Runge VM, Henkelman RM. Overcoming motion in abdominal MR imaging. AJR 1988; 150:513-522.

17. Jung G, Krahe T, Kugel H, et al. Prospective comparison of fast SE and GRASE sequences and echo planar imaging with conventional SE sequences in the detection of focal liver lesions at $1.0 \mathrm{~T}$. J Comput Assist Tomogr 1997;21:341-347.

18. Allkemper T, Reimer P, Schuierer G, Peters PE. Study of susceptibility-induced artifacts in GRASE with different echo train length. Eur Radiol 1998;8: 834-838.

19. Hood MN, Ho VB, Smirniotopoulos JG, Szumowski J. Chemical shift: the artifact and clinical tool revisited. RadioGraphics 1999;19:357-371.

20. Mirowitz SA. MR imaging artifacts. Challenges and solutions. Magn Reson Imaging Clin N Am 1999; 7 717-732. 